\title{
A Fast Mode Decision Algorithm for Intra Prediction in HEVC
}

\author{
Li Wei, Wang Rangding, Wang Jiaji, Xu Dawen, Xu Jian \\ CKC Software Lab \\ Ningbo University \\ Ningbo, China \\ e-mail: wangrangding@nbu.edu.cn
}

\begin{abstract}
To reduce the intra encoding complexity of HEVC, a fast intra prediction mode decision algorithm is proposed in this paper. According to different size of prediction unit, based on statistical data, the algorithm uses threshold method to exclude the intra prediction modes of small possibility, then reduce the number of intra prediction modes for the rate distortion cost (RD Cost) calculation and cut down the encoding complexity. Experimental results show that compared with the HEVC reference software HM12.0, the proposed algorithm can achieve a reduction of $24.27 \%$ in average in intra prediction coding time, with a negligible average PSNR loss and average bit rate increment.

Key words: HEVC; intra prediction; statistical results; mode selection
\end{abstract}

\section{INTRODUCTION}

The High Efficiency Video Coding (HEVC)[1] is developed by the Joint Collaborative Team on Video Coding(JCT-VC) jointly established by ISO/IEC MPEG and ITU-T VCEG in April 2010. It is mainly aimed at the application of the high resolution and 3-D video. HEVC has become a new generation of international video coding standard in January 2013. Its main objective is to provide two times compression efficiency of H.264/AVC [2]. That is to say the bit rate is reduced by $50 \%$ while keeping equal perceptual quality compared to H.264/AVC.

HECV also adopts the same hybrid video coding framework as H.264/AVC, but it has significantly improved in terms of intra coding. HEVC intra coding unit adopts recursive splitting technology based on the quad-tree structure and multi-angle intra prediction modes selection technology [3]. There are three basic units-Coding Unit (CU), Prediction Unit (PU) and Transform Unit (TU) [4]. HEVC selects the optimal intra prediction mode from all of the 35 intra prediction modes according to the RD Cost. The separation of three basic units and introduction of the multiangle of intra prediction mode technology make it more flexible and precise in prediction, transform and compress coding. The effect of compressing are more fit for the characteristics of the video image itself. At the same time, it also brings high encoding complexity and increases the coding time which are bad for HEVC to be used in the realtime video applications. Therefore, reducing the complexity of intra prediction without affecting the performance of HEVC coding has become a hot issue.
In the development of HEVC, the research of fast intra coding algorithm focuses on two aspects: one is the termination and skip ahead of recursive quad-tree coding unit dividing; The other is the quick selection of optimal intra prediction mode. Shen et al. [5] adopts the way of both time domain prediction and spatial domain prediction, using weighted depth value of the LCU (Large Coding Unit) in the same location of previous frame and the left and above adjacent position to predict the depth value of the current LCU, then skip or terminate ahead the corresponding depth. Jiang et al. [6] utilizes the threshold value which is concerned with depth and RD Cost to detect the smoothness of current video and skips the process of coding unit. For the selection of optimal intra prediction mode, reference[7] proposed the concept of rough mode decision(RMD) for the first time, utilized the cost based on SATD to choose a few prediction modes in the early stage and finally did the RD Cost calculation of prediction modes. Reference [8], as the supplement of reference [7], proposed the concept of most probable mode (MPM) and did the RD Cost calculation of the modified RMD and MPM, so this simplify intra prediction mode selection greatly. Reference [9] proposed an early termination method based on hierarchical structure to judge the current PU whether needs to be further divided, at the same time the number of candidates of RMD is reduced and the optimal intra prediction mode of corresponding previous-depth prediction unit is included in the process of RD Cost calculation.

The algorithms above did not make full use of the statistical properties of prediction modes and cost based on SATD after RMD. A fast intra prediction mode decision algorithm is proposed in this paper. For prediction units of large size $(64 \times 64,32 \times 32,16 \times 16)$, this paper adopts simple threshold value method to reduce the number of prediction modes after RMD to 1 or 2 . For prediction units of small size, $(8 \times 8,4 x 4)$, this paper analyses its distribution of cost based on SATD after RMD and reduce the number of prediction modes under two conditions.

The rest of this paper is organized as follows: Section II will introduce HEVC intra coding. The proposed fast intra prediction mode decision algorithm is presented in Section III. Section IV demonstrates experimental results and discussion. Section V concludes this paper. 


\section{INTRA- CODING COMPLEXITY ANALYSIS IN HEVC}

\section{A. Intra-coding}

The principle of intra coding in HEVC follows the technology of H.264/AVC. By using the spatial correlation of pixels, the pixel value of current macro-blocks was predicted by the boundary pixel value of adjacent coded and reconstructed macro-blocks [10]. The optimal intra prediction mode is the mode which has the minimum RD Cost by iterating all of the 35 intra prediction modes. Compared with H.264/AVC, which used the fixed size macro-block, HEVC uses larger CU from $64 \times 64$ to $8 \times 8$. Each frame of video is divided into LCU, however, the LCU is further divided following a quad-tree block structure, until small coding unit (SCU). H.264/AVC has only 9 intra prediction modes, as shown in Fig.1(a).HEVC enriches the number of intra prediction modes up to 35 including 33 angle prediction modes, DC mode and Planar mode, as shown in Fig.1(b)[11].

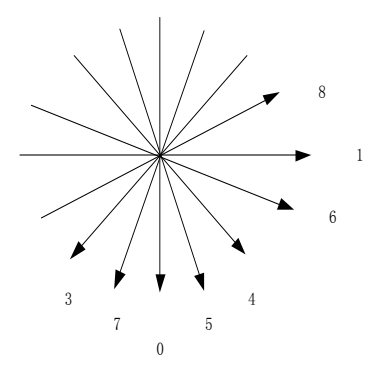

(a)

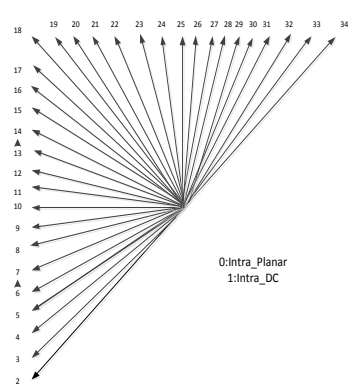

(b)
Figure1. Prediction modes in H.264/AVC and HEVC

\section{B. Intra Prediction Mode Selection}

Firstly, RMD is proposed to choose candidates from all the 35 modes shown in Fig.1 (b).RMD tests the sum of the absolute transform coefficient differences (SATD) and sort the modes in the ascending order. Table I illustrates the number of modes for RMD.

Secondly, the MPMs which obtained from left and above PUs are adopted as candidate modes

Finally, HEVC calculates RD Cost of every intra prediction mode in the candidate list and the minimum RD Cost mode is the optimal intra prediction mode.

TABLE I. NUMBER OF INTRA PREDICTION MODE FOR PU

\begin{tabular}{|c|c|c|c|c|c|}
\hline PU Size & $64 \times 64$ & $32 \times 32$ & $16 \times 16$ & $8 \times 8$ & $4 \times 4$ \\
\hline NO. For RMD & 3 & 3 & 3 & 8 & 8 \\
\hline
\end{tabular}

\section{Intra -coding Complexity Analysis}

In HEVC, taking a LCU for example, the determination of coding structure needs recursive traversal from depth 0 to 3 so that HEVC can get various sizes of CUs. For each CU, HEVC needs to calculate the cost of prediction modes corresponding to the PU except $8 \times 8 \mathrm{CU}$. It also needs to do mode selection for $4 \times 4$ PU when the size of $\mathrm{CU}$ is $8 \times 8$. Table II shows the number of the PU in a LCU. TABLE II. NUMBER OF PU IN A LCU

\begin{tabular}{|c|c|c|c|c|c|}
\hline PU Size & $64 \times 64$ & $32 \times 32$ & $16 \times 16$ & $8 \times 8$ & $4 \times 4$ \\
\hline NO. Of PU & 1 & 4 & 16 & 64 & 256 \\
\hline
\end{tabular}

For the luminance component, HEVC needs to calculate 11935 times cost based on SATD. Without consideration of MPM, HEVC needs to calculate 2623 times RD Cost of intra prediction mode. Even the efficiency and accuracy were improved, though the calculation is very large and complexity of the algorithm is very high. Therefore, the key to decrease the coding time is to reduce the times of calculating RD Cost.

\section{FAST INTRA PREDICTION MODE DECISION OF HEVC}

According to different size of PU, based on statistical data of intra prediction modes and cost, the algorithm uses threshold method to reduce the number of intra prediction modes for the RD Cost calculation and cut down the encoding time.

\section{A. Statistical Analysis of Prediction Modes}

The number of prediction mode after RMD for PU of large size $(64 \times 64,32 \times 32,16 \times 16)$ is three. For five kinds of different resolution video test sequences, this paper remains the first prediction mode plus MPM, the first two prediction modes plus MPM and MPM. Under three cases above, we get the hit rate of the optimal prediction mode, the average hit rate is showed in Fig.2. The horizontal axis represents the size of PU, vertical axis represents average hit rate.

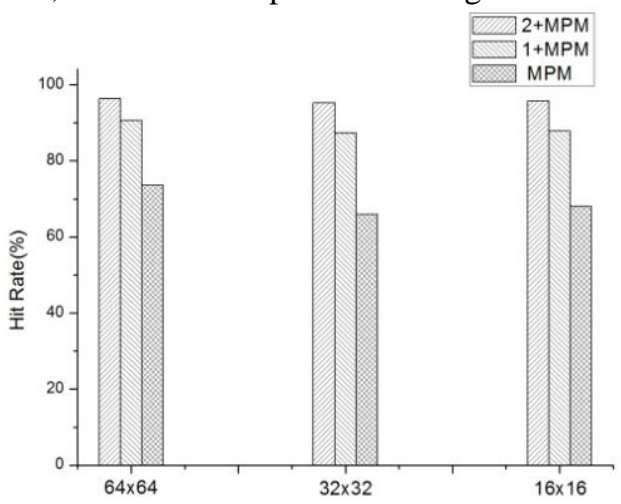

Figure2. Hit rate of the optimal prediction modes for large size

The number of prediction mode after RMD for PU of small size $(8 \times 8,4 \times 4)$ is eight. This paper remains the first three prediction modes plus MPM, the first four prediction modes plus MPM, the first five prediction modes plus MPM and the first six prediction modes plus MPM. Under four cases above, we get the hit rate of the optimal prediction mode, the average hit rate is showed in Fig.3. 


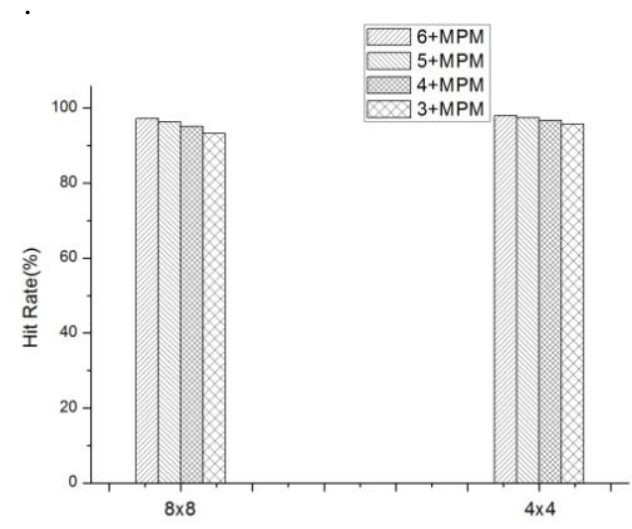

Figure3. Hit rate of the optimal prediction modes for small size

We can have a conclusion that the hit rate can reach $87 \%$ if we remain the first prediction mode after RMD plus MPM for large size. When the number of prediction mode after RMD is two, the corresponding hit rate can reach $95 \%$. For PU of small size, the hit rate can reach $93 \%$ even we remain the first three prediction modes plus MPM. That is to say, the number of prediction modes after RMD can be reduced further. There is no need to select the optimal intra prediction mode from the determined number of intra prediction modes after RMD. We can exclude some prediction modes with small possibility by a certain amount of calculation, then reduce the number of intra prediction modes for the RD Cost calculation and cut down the intracoding time.

\section{B. Statistical Analysis of Cost Based on SATD}

SATD is the Hardamard transform of prediction residual. A good prediction mode should produce a small value of SATD. The cost based on SATD can reflect distortion and bit rate. The cost function based on SATD is showed in type (1).

$$
\cos t=S A T D+\sqrt{\lambda} \bullet B_{\text {pred }}
$$

Where $B_{\text {pred }}$ denotes the bit rate when HEVC uses the current intra prediction mode and $\lambda$ is the Largrange multiplier.

This paper analyzes the eight costs of prediction modes after RMD and records the probability of same cost. The statistical result is showed in table III and table IV.

\section{TABLE III THE PROBABILITY OF SAME COST}

\begin{tabular}{|c|c|c|c|c|c|c|c|c|}
\hline & \multicolumn{7}{|c|}{ The Number of Same Cost For 8x8 PU } \\
\hline Resolution & $\mathbf{1}$ & $\mathbf{2}$ & $\mathbf{3}$ & $\mathbf{4}$ & $\mathbf{5}$ & $\mathbf{6}$ & $\mathbf{7}$ & $\boldsymbol{8}$ \\
\hline $\mathbf{4 1 6 x \mathbf { 4 0 }}$ & 67.05 & 20.73 & 3.84 & 2.46 & 4.08 & 1.13 & 0.53 & 0.17 \\
\hline $\mathbf{8 3 2 x 4 8 0}$ & 71.12 & 20.88 & 3.51 & 1.49 & 2.17 & 0.54 & 0.23 & 0.07 \\
\hline $\mathbf{1 2 8 0 x 7 2 0}$ & 37.48 & 22.04 & 7.73 & 5.18 & 26.79 & 0.48 & 0.21 & 0.09 \\
\hline $\mathbf{1 9 2 0 x \mathbf { 1 0 8 0 }}$ & 54.97 & 28.94 & 6.06 & 3.08 & 5.74 & 0.72 & 0.34 & 0.16 \\
\hline $\mathbf{2 5 6 0 x \mathbf { 1 6 0 0 }}$ & 66.24 & 20.48 & 4.14 & 2.58 & 5.21 & 0.84 & 0.37 & 0.14 \\
\hline
\end{tabular}


algorithm is developed with VS2010 on a 64-bit computer with Inter(R)-Core(TM) i3-2130 3.4GHz CPU.

The main reference software coding configuration parameters are shown in Table $\mathrm{V}$, and the remaining parameters are set to the default configuration.

TABLE V CONFIGURATION PARAMETERS OF THE HM

\begin{tabular}{cc}
\hline Parameters & Configuration \\
\hline Frames To Be Encoded & 1 \\
Intra Period & 1 \\
QP & 2273237 \\
GOP Size & 8 \\
Max Depth & 4 \\
\hline
\end{tabular}

Experiments also evaluate algorithm from the objective video coding quality variation $(\triangle \mathrm{PSNR})$, bit rate variation $(\triangle \mathrm{BR})$ and the percentage change in coding time $(\Delta \mathrm{T})$. The formulas are described as following:

$$
\begin{aligned}
& \Delta P S N R=P S N R_{P R O}-P S N R_{H M} \\
& \Delta B R=\frac{B R_{P R O}-B R_{H M}}{B R_{H M}} \times 100 \% \\
& \Delta T=\frac{T_{P R O}-T_{H M}}{T_{H M}} \times 100 \%
\end{aligned}
$$

TABLE VI. EXPERIMENTAL RESULTS

\begin{tabular}{c|c|c|c|c}
\hline Test Sequences & QP & $\Delta \mathrm{BR}(\%)$ & $\Delta \mathrm{PSNR}(\mathrm{dB})$ & $\Delta \mathrm{T}(\%)$ \\
\hline \multirow{4}{*}{ BasketballPass_416x240_50 } & 22 & -0.139 & -0.0673 & -27.91 \\
\cline { 2 - 5 } & 27 & -0.364 & -0.0585 & -25.74 \\
\cline { 2 - 5 } & 32 & -0.212 & -0.0523 & -22.25 \\
\cline { 2 - 5 } & 37 & -0.653 & -0.0614 & -24.48 \\
\hline \multirow{4}{*}{ BasketballDrill_832x480_50 } & 22 & -0.336 & -0.0573 & -28.44 \\
\cline { 2 - 5 } & 27 & -0.333 & -0.0458 & -23.41 \\
\cline { 2 - 5 } & 32 & -0.276 & -0.0349 & -22.62 \\
\cline { 2 - 5 } & 37 & -0.385 & -0.0219 & -21.08 \\
\hline \multirow{4}{*}{ Johnny_1280x720_60 } & 22 & -0.045 & -0.0349 & -23.81 \\
\cline { 2 - 5 } & 27 & +0.148 & -0.0325 & -22.19 \\
\cline { 2 - 5 } & 32 & +0.248 & -0.0316 & -24.57 \\
\cline { 2 - 5 } & 37 & +0.162 & -0.0258 & -24.24 \\
\hline \multirow{4}{*}{ Cactus_1920x1080_50 } & 22 & -0.356 & -0.0470 & -20.69 \\
\cline { 2 - 5 } & 27 & -0.109 & -0.0295 & -31.79 \\
\cline { 2 - 5 } & 32 & -0.164 & -0.0383 & -27.28 \\
\cline { 2 - 5 } & 37 & +0.068 & -0.0273 & -20.52 \\
\hline \multirow{4}{*}{ average } & 22 & -0.062 & -0.0653 & -23.64 \\
\cline { 2 - 5 } & 27 & +0.071 & -0.0509 & -26.94 \\
\cline { 2 - 5 } & 32 & -0.044 & -0.0490 & -19.06 \\
\cline { 2 - 5 } & 37 & -0.014 & -0.0363 & -24.66 \\
\hline
\end{tabular}

As is showed in table VI, compared with the original algorithm in HM12.0, the proposed algorithm can reduce average about $24.27 \%$ in coding time, the bit rate is slightly decreased and the PSNR is dropped by 0.0434 which can be neglected. The quality of coding is approximate constant. We have a conclusion that the algorithm what we proposed is effective to different resolution video.

\section{CONCLUSIONS}

In this paper, a fast intra prediction mode decision algorithm is proposed, which is based on statistical analysis of prediction modes and cost value after RMD. The algorithm can exclude some prediction modes of small possibility, reduce the number of intra prediction modes for $\mathrm{t}$ RD Cost calculation and save the encoding time. Experimental results show that compared with the reference software HM12.0, the average reduction of intra prediction coding time is $24.27 \%$, while the code performance basically remain unchanged. What's more, the algorithm for video sequences with different resolutions all has a certain effect and the realization of the algorithm is simple.

\section{ACKNOWLEDGMENT}

This work is sponsored by K.C. Wong Magna Fund in Ningbo University, National Natural Science Foundation of China (NSFC: 61170137, 61300055, 61301247), Doctoral Fund of Ministry of Education of China(20103305110002), Zhejiang natural science foundation of China (ZJNSF: LY13F020013), Ningbo natural science foundation of China (2013A610057, 2013A610059), Scientific Research Fund of Zhejiang Provincial Education Department (Y201119434), Open Fund of Zhejiang Provincial Top key Discipline of Information and Communication Engineering(XKXL1313, XKXL1310).

\section{REFERENCES}

[1] Han G J, Ohm J R, Han W J, "Overview of the high efficiency video coding (HEVC) standard," vol22, 2012, pp. 1649-1668.

[2] Wiegand T, Sullivan G J, Bjontegaard G, et al. "Overview of the $\mathrm{H}$. 264/AVC video coding standard" Circuits and Systems for Video Technology, IEEE Transactions . vol. 13, 2003, pp. 560-576.

[3] Jani L, Frank B, Woo-Jin H, et al. "Intra Coding of the HEVC Standard" IEEE Transactions on Circuits and Systems for Video Technology, vol.12, 2012, pp. 1792-1802.

[4] Kim I K, Min J, Lee T, et al. "Block partitioning structure in the HEVC standard". Circuits and Systems for Video Technology, IEEE Transactions on, vol. 22, 2012, pp. 1697-1706.

[5] Shen L Q, Liu Z, Zhang X P, et al. "An Effective CU Size Decision Method for HEVC Encoders". IEEE Transactions on Multimedia, vol.15, 2013, pp. 465-470.

[6] Jiang J, Guo B L, et al."Fast intra coding algorithm using smooth region detection for HEVC", Journal of Xidian University, vol. 3, 2013, pp. 194-200.

[7] Piao Y, Min J, Chen J. "Encoder improvement of unified intra prediction"JCTVC-C207, Guangzhou, China, Oct, 2010, unpublished.

[8] Zhao L, Zhang L, Ma S, et al. " Fast mode decision algorithm for intra prediction in HEVC", Visual Communications and Image Processing (VCIP), 2011 IEEE (pp. 1-4).IEEE.

[9] Kim J, Yang J, Lee H. "Fast intra mode decision of HEVC based on hierarchical structure", Information, Communications and Signal Processing (ICICS) 2011 8th International Conference on (pp. 14).IEEE.

[10] Wang H, K Wong S, Kok C W. "An efficient mode decision algorithm for H.264/AVC encoding optimization". Multimedia, IEEE Transactions on, vol. 9, April, 2007, pp. 882-888.

[11] Lainema J, Ugur K. "Angular intra prediction in high efficiency video coding (HEVC)”, Multimedia Signal Processing (MMSP), 2011 IEEE 13th International Workshop on (pp.1-5).IEEE. 